\title{
Focal Adhesion Kinase Anchoring Kinetics and Regulatory Interactions Quantified by Total Internal Reflection Fluorescence Microscopy
}

\author{
Taylor Zak ${ }^{1}$, Allen M. Samarel ${ }^{1,2}$ and Seth L. Robia ${ }^{1}$ \\ ${ }^{1}$ Department of Cell and Molecular Physiology, Stritch School of Medicine, Loyola University Chicago, \\ Maywood, IL USA \\ ${ }^{2}$ Department of Medicine, Stritch School of Medicine, Loyola University Chicago, Maywood, IL USA
}

The ubiquitously expressed focal adhesion kinase (FAK) plays a central role in modulating vascular smooth muscle cell (VSMC) migration. FAK-Related Non-Kinase (FRNK), the autonomously expressed C-terminal domain of FAK, is a VSMC-specific protein whose expression is greatly increased in injured arteries, and serves to inhibit growth and migration.[1] We have previously shown that FRNK inhibits FAK-dependent VSMC signaling and migration in cell culture and in vivo.

To investigate molecular mechanisms of FAK regulation by FRNK, we quantified intermolecular FRET with acceptor-selective photobleaching. We observed FRET from FAK to FAK and FRNK to FRNK (homo-oligomers) and from FAK to FRNK (heterooligomeric regulatory complex) (Fig. 1). For all complexes we observed very little FRET in the cytoplasm (Fig. 1), consistent with another group's previous studies of FAK-FAK binding [2]. To investigate the stoichiometry of these regulatory complexes, we performed progressive photobleaching FRET, which reveals whether the regulatory complex is a dimer or a higher order oligomer [3]. The data suggest a highly heterogeneous population of FAs, with differential FRET efficiency and stoichiometry observed for different FAs within the same VSMC. The data suggest that FAK forms predominantly dimers[2], but some FAs show evidence of higher order oligomers [4]. To investigate the kinetics of FAK/FRNK anchoring to FAs we performed single-molecule TIRF microscopy of YFP-FAK/FRNK in live cells (Fig. 2A). This image is the simple summation of all single molecule events in a series of 10,000 images, and panels B-D represent an enlargement of the 7.5 micron box shown in panel A. Fig. 2B reveals the typical diffraction-limit resolution obtained by conventional TIRF imaging. A single frame of the image series (Fig. 2C) reveals 4 discrete single molecules of FRNK visible in the region as Gaussian-shaped spots. Single molecule transients were fit with the ImageJ plugin QuickPALM to obtain super-resolution maps of all of the FRNK anchoring events (Fig. 2D). We quantified the fluorescence transients to observe single molecule binding/unbinding events and quantify the kinetics of these events. Single molecule fluorescence trajectories (Fig. 2E) were characterized by brief fluorescence bursts of uniform intensity flanked by periods of dark when no molecules were present. Histogram analysis (Fig. 2F) reveals two major Gaussian peaks corresponding to background ("0") and bright FRNK monomer binding events (" $1 \mathrm{X}$ ”). Events with approximately 2-fold brighter fluorescence intensity were only rarely observed (" $2 \mathrm{X}$ ”), suggesting FRNK anchors in FAs as a monomeric species. We obtained the average decay profile of a fluorescence burst (Fig. 2G) and performed dwell time analysis. A histogram of dwell times revealed a multiphasic distribution, with many short binding events $(<50 \mathrm{~ms})$ and another smaller population of longer dwell-time binding events (>200 ms) (Fig. 2H).

Overall the data are compatible with the hypothesis that regulatory interactions of FAK and FRNK control the dynamics of FAK anchoring in focal adhesions, a key determinant of cell growth and migration. Future experiments will determine the role of structural mutations and phosphorylation in tuning FAK/FRNK anchoring kinetics. 


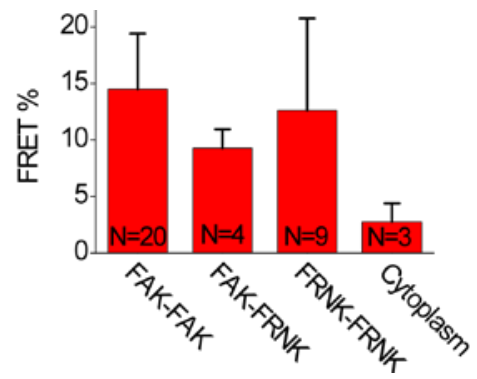

Fig. 1. FRET evidence of FAK-FAK and FRNK-FRNK homo-oligomers as well as a heterodimer species. FRET values were obtained from focal adhesions and very little FRET was observed in the cytoplasm.
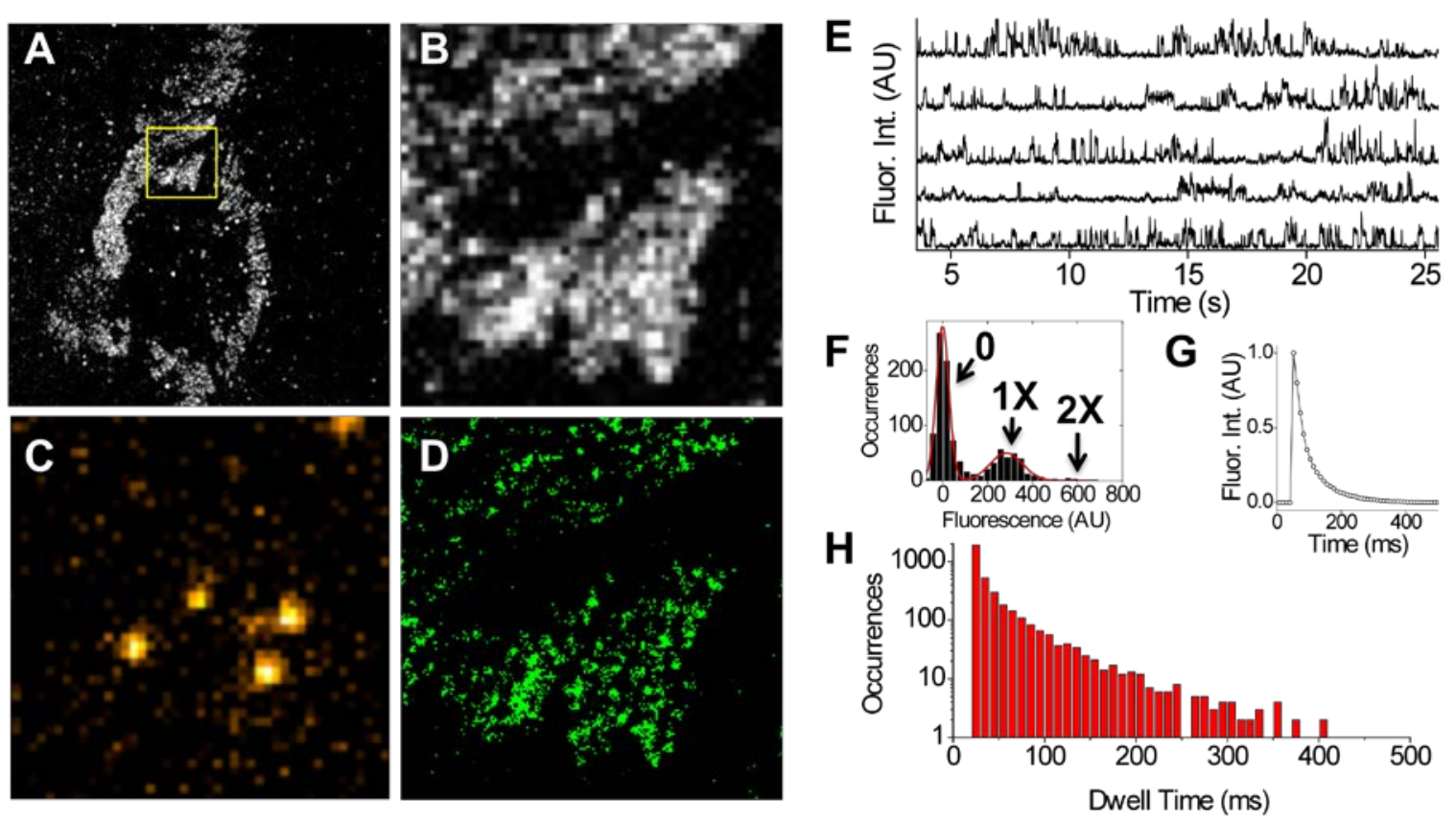

Fig. 2. Single molecule fluorescence studies of FRNK anchoring. A) TIRF microscopy sum image. Boxed region is $7.5 \times 7.5 \mu \mathrm{m} \mathrm{B}$ ) Enlarged view of boxed region C) Image of single molecules of YFPFRNK observed in boxed region. D) Super-resolution map of FRNK anchoring events in boxed region E) Fluorescence bursts indicating single molecule anchoring events. F) A histogram of measured fluorescence values consistent with a single species. G) Avg. of 3000 fluorescence bursts. H) Dwelltime analysis of anchoring events reveals the persistence of FRNK at anchoring sites.

\section{References:}

[1] Koshman YE, Engman SJ, Kim T, Iyengar R, Henderson KK, Samarel AM. Role of FRNK tyrosine phosphorylation in vascular smooth muscle spreading and migration. Cardiovasc Res. 2010;85:571-81.

[2] Brami-Cherrier K, Gervasi N, Arsenieva D, Walkiewicz K, Boutterin MC, Ortega A, et al. FAK dimerization controls its kinase-dependent functions at focal adhesions. EMBO J. 2014;33:356-70.

[3] Kelly EM, Hou Z, Bossuyt J, Bers DM, Robia SL. Phospholamban oligomerization, quaternary structure, and sarco(endo)plasmic reticulum calcium ATPase binding measured by fluorescence resonance energy transfer in living cells. The Journal of biological chemistry. 2008;283:12202-11.

[4] Goni GM, Epifano C, Boskovic J, Camacho-Artacho M, Zhou J, Bronowska A, et al. Phosphatidylinositol 4,5-bisphosphate triggers activation of focal adhesion kinase by inducing clustering and conformational changes. Proc Natl Acad Sci U S A. 2014;111:E3177-86.

[5] The authors acknowledge funding from NIH (HL092321, HL062426). 